\title{
POSITIVITY OF GLOBAL BRANCHES OF FULLY NONLINEAR ELLIPTIC BOUNDARY VALUE PROBLEMS
}

\author{
TIMOTHY J. HEALEY AND HANSJÖRG KIELHÖFER
}

(Communicated by Barbara L. Keyfitz)

\begin{abstract}
We consider a bifurcation problem for a general class of fully nonlinear, second-order elliptic equations on a regular bounded domain in $\mathbb{R}^{n}$ and subject to homogeneous Dirichlet boundary data. We assume that the linearized problem about the trivial solution possesses a positive solution for at least one isolated parameter value. With no other growth or sign conditions imposed upon the nonlinearity, we establish the existence of a global branch of nontrivial positive solutions. Moreover, if there is only one such isolated value of the parameter, we deduce that the branch of positive solutions is unbounded.
\end{abstract}

\section{INTRODUCTION}

The model equation,

$$
\begin{aligned}
\Delta u+\lambda f(u)=0 & \text { in } \Omega \subset \mathbb{R}^{n}, \lambda \in \mathbb{R}, \\
u=0 & \text { on } \partial \Omega, \\
u>0 & \text { in } \Omega,
\end{aligned}
$$

has been studied by many authors; we refer to the reviews $[1,9]$ and the monograph [12] where many additional references can be found. Besides growth conditions on $f$ near infinity, an essential assumption in most contributions is that the function $f$ is nonnegative on $\mathbb{R}_{+}$. References $[7,10]$ are exceptional in this respect in that no sign condition is imposed upon the nonlinearity. Their methods, however, do not allow for the dependence of $f$ on the gradient of $u$; by adding (and subtracting) some $\lambda c u>0$, they make $f$ nonnegative in order to apply a minimum principle and a Hopf boundary lemma. The results in [10] appear to be the most general, applying to quasi-linear equations. (We do not deny that in many papers the goal is beyond mere existence of positive branches; the number of positive solutions and the qualitative analysis of bifurcation diagrams are of special interest.)

Here we present a result for a class of nonlinear, elliptic problems over a

Received by the editors November 26, 1990 and, in revised form, January 22, 1991.

1991 Mathematics Subject Classification. Primary 35B32, 35B50, 35J65. 
regular domain of the form

$$
\begin{aligned}
F\left(\nabla^{2} u, \nabla u, u, \mathbf{x}, \lambda\right)=0 & \text { in } \Omega \subset \mathbb{R}^{n}, \lambda \in \mathbb{R}, \\
u=0 & \text { on } \partial \Omega, \\
u>0 & \text { on } \Omega .
\end{aligned}
$$

Assuming $F(0,0,0, \mathbf{x}, \lambda)=0$, we have the trivial solution $(\lambda, u)=(\lambda, 0)$ for all $\lambda \in \mathbb{R}$, and we assume that the linearized problem has a positive "eigenfunction" spanning a one-dimensional kernel for some isolated value of the parameter $\lambda=\lambda_{0}$. For a large class of problems, such a positive eigenfunction is guaranteed by the Krein-Rutman Theorem. We emphasize that, due to the generalization in [7], the zero-order coefficient $F_{u}(0,0,0, \mathbf{x}, \lambda)$ need not be definite in order to obtain a positive eigenfunction. The existence of global bifurcating branches of solutions of fully nonlinear elliptic problems was recently established in [3]. The basic idea is first to differentiate the equation, thus yielding a higher-order quasilinear problem, for which recent generalizations of Rabinowitz's classical theorem [10] are applicable, cf. [3, 8]. In $\S 2$ we summarize the relevant steps leading to the existence of a global branch (continuum) of solutions of $(1.2)_{1,2}$. Our main contribution is presented in $\S 3$; with no growth or sign conditions imposed upon $F$, we show that all solutions contained in the branch constructed in $\S 2$ are positive (satisfy $(1.2)_{3}$ ). The basic tool here is a minimum principle, as given in $[4,11]$, for linear equations. If $\lambda_{0}$ happens to be the only parameter value for which the linearized problem admits a positive eigenfunction, we immediately conclude that our positive global solution branch is unbounded in some appropriate norm.

\section{Global bifurcation}

Let $\Omega \subset \mathbb{R}^{n}$ be any bounded domain with a boundary $\partial \Omega$ of class $C^{2+\alpha}$, $0<\alpha<1$. We study the parameter-dependent boundary value problem,

$$
\begin{aligned}
F\left(\nabla^{2} u, \nabla u, u, \mathbf{x}, \lambda\right) & =0 & & \text { in } \Omega, \\
u & =0 & & \text { on } \partial \Omega,
\end{aligned}
$$

where $F$ is a $C^{3}$-function of its arguments, $\nabla u$ denotes the gradient with components $u_{x_{i}}$, and $\nabla^{2} u$ denotes the second gradient or Hessian with components $u_{x_{i} x_{j}}, i, j=1, \ldots, n$. (Here and in the sequel we use summation convention, and $u_{x_{i}} \equiv \partial u / \partial x_{i}, u_{x_{i} x_{j}}=\partial^{2} u / \partial x_{i} \partial x_{j}$.) For $F$ we assume uniform ellipticity:

$$
\alpha|\xi|^{2} \leq F_{w_{i j}}(\mathbf{W}, \mathbf{v}, u, \mathbf{x}, \lambda) \xi_{i} \xi_{j} \leq \beta|\xi|^{2} \text { for all }(\mathbf{W}, \mathbf{v}, u, \mathbf{x}, \lambda)
$$
$\in \mathbb{R}_{\text {sym }}^{n \times n} \times \mathbb{R}^{n} \times \mathbb{R} \times \bar{\Omega} \times \mathbb{R}, \xi \in \mathbb{R}^{n}$. The positive constants $\alpha, \beta$ are uniform on bounded subsets of $\mathbb{R}_{\mathrm{sym}}^{n \times n} \times \mathbb{R}^{n} \times \mathbb{R} \times \bar{\Omega} \times \mathbb{R}$.

Assuming $F(0,0,0, \mathbf{x}, \lambda)=0$ for all $(\mathbf{x}, \lambda) \in \bar{\Omega} \times \mathbb{R}$, we have the trivial solution $(\lambda, u)=(\lambda, 0)$ for all $\lambda \in \mathbb{R}$. For bifurcation from this trivial branch, we study the linearization

$$
\begin{aligned}
& F_{w_{i j}}(0,0,0, \mathbf{x}, \lambda) u_{x_{i} x_{j}}+F_{v_{i}}(0,0,0, \mathbf{x}, \lambda) u_{x_{i}}+F_{u}(0,0,0, \mathbf{x}, \lambda) u \\
& =0 \text { in } \Omega, \text { together with the boundary condition } u=0 \text { on } \partial \Omega .
\end{aligned}
$$

By (2.2) this is a linear elliptic boundary value problem. A necessary condition for bifurcation at some $\left(\lambda_{0}, 0\right)$ is that $(2.3)$ have a nontrivial solution for $\lambda_{0} \in$ $\mathbb{R}$. If the parameter $\lambda$ occurs only linearly in the zero-order term, then $(2.3)$ is 
an eigenvalue problem in the classical sense. It is not the goal of this work to study the linear problem (2.3) in its generality, but we simply assume that,

for some $\lambda_{0} \in \mathbb{R}$, the linear operator defined by the left side of (2.3) has a one-dimensional kernel spanned by a function $u_{0}$ that is positive in $\Omega$, and a closed range of codimension one.

For a large class of problems, the Krein-Rutman Theorem guarantees the existence of $u_{0}$ (e.g. see [12]). For a generalization of the classical results, we refer to [7].

The functional analytic setting of $(2.1)$ is described as follows: For $k \in$ $\mathbb{N} \cup\{0\}$, let

$C^{k+\alpha}(\bar{\Omega})$ be the usual Hölder spaces with norm \|\|$_{k+\alpha}, E=$ $C^{\alpha}(\overline{\mathbf{\Omega}}), D=C^{2+\alpha}(\bar{\Omega}) \cap\left\{\left.u\right|_{\partial \Omega}=0\right\}$.

Then the left-side of (2.1) defines a mapping

$G: \mathbb{R} \times D \rightarrow E$, which is twice continuously Fréchet differentiable.

The first-order derivative $G_{u}(\lambda, u): D \rightarrow E$ is of the form

$$
\begin{aligned}
G_{u}(\lambda, u) h= & F_{w_{i j}}\left(\nabla^{2} u, \nabla u, \mathbf{x}, \lambda\right) h_{x_{i} x_{j}}+F_{v_{i}}\left(\nabla^{2} u, \nabla u, u, \mathbf{x}, \lambda\right) h_{x_{i}} \\
& +F_{u}\left(\nabla^{2} u, \nabla u, \mathbf{x}, \lambda\right) h \quad \text { for }(\lambda, u) \in \mathbb{R} \times D, h \in D,
\end{aligned}
$$

By virtue of (2.2), $G_{u}(\lambda, u)$ is a uniformly elliptic operator for each $(\lambda, u) \in$ $\mathbb{R} \times D$. The Schauder estimate then implies that $G_{u}(\lambda, u)$ is semi-Fredholm. By assumption (2.4), $G_{u}\left(\lambda_{0}, 0\right)$ is Fredholm of index zero. Thus, $G_{u}(\lambda, u)$ has index zero, by the stability of the Fredholm index. Furthermore, it is well known that the operator $G_{u}(\lambda, u)$ is sectorial and that its spectrum consists of isolated eigenvalues of finite (algebraic) multiplicity. (For more details and a reference, see [5] and [6].) Therefore, by (2.4),

zero is an isolated eigenvalue of finite (algebraic) multiplicity of the Fredholm operator $G_{u}\left(\lambda_{0}, 0\right): D \rightarrow E$, where $G_{u}\left(\lambda_{0}, 0\right)$ is given by the left side of (2.3).

Therefore, the crossing number $\chi\left(\lambda_{0}\right)$ through 0 at $\lambda=\lambda_{0}$ is defined for the family $G_{u}(\lambda, 0)$ (see [8]), and we assume that

$$
\chi\left(\lambda_{0}\right) \text { is odd. }
$$

$\left(\chi\left(\lambda_{0}\right)\right.$ is the number of eigenvalues (counting multiplicities) in the 0 -group of the eigenvalue perturbation of $G_{u}(\lambda, 0)$ that leave the left complex halfplane through 0 when the parameter $\lambda$ passes through $\lambda_{0}$.)

We emphasize that if the Krein-Rutman Theorem is applicable (including the generalization given in [7]), then the zero eigenvalue of $G_{u}\left(\lambda_{0}, 0\right)$ is simple, and therefore $\chi\left(\lambda_{0}\right)= \pm 1$. In the case of "simplicity in the sense of CrandallRabinowitz" [2], their nondegeneracy condition is equivalent to an odd crossing number (see [8]). We do not pursue the problem of odd crossing numbers for $G_{u}(\lambda, 0)$ near $\lambda=\lambda_{0}$ in its generality. In particular cases, e.g., [6], $\chi\left(\lambda_{0}\right)$ can be computed explicitly.

As shown in [8], an odd crossing number implies local bifurcation. Therefore, the component $\Sigma_{0} \subset \mathbb{R} \times D$ in the closure of the set of nontrivial solutions of $G(\lambda, u)=0$ that contains $\left(\lambda_{0}, 0\right)$ is not empty. 
We call the component $\Sigma_{0}$ global, which we now describe in more detail. Since $F$ is fully nonlinear, it does not necessarily define a proper map $G$ (2.6). Therefore we do not know if $\Sigma_{0}$ is subject to the Rabinowitz alternative in $\mathbb{R} \times D$ (see [10] or [8]). There is a way, however, to give a weaker alternative for $\Sigma_{0}$. Following [3], we convert equations (2.1) into a quasi-linear elliptic boundary value problem of fourth order that has precisely the same solution set as (2.1). As shown in [10] or [8], quasi-linear elliptic operators are proper (and admissible in the sense of [8]; see [6]), and therefore global bifurcating continua are subject to the Rabinowitz alternative, which, in our particular case, reads as follows:

Let $F$ be a $C^{5}$-function of its arguments. Then $\Sigma_{0}$, emanating at $\left(\lambda_{0}, 0\right)$, is unbounded in $\mathbb{R} \times C^{4+\alpha}(\bar{\Omega})$ or it meets the trivial solution set at some different $\left(\lambda_{1}, 0\right)$.

Remark. There is a price to pay for the above conclusion and also for the more general results of [3], which is not explicitly discussed in that work; namely, the alternative $(2.11)$ does not exclude that $\Sigma_{0} \cap(\mathbb{R} \times\{0\})=\left(\lambda_{0}, 0\right)$ and $\Sigma_{0}$ is bounded in $\mathbb{R} \times D$. If (1.2) is quasi-linear, however, then the $C^{4+\alpha}(\bar{\Omega})$ topology in $(2.11)$ can be replaced by the $C^{1+\alpha}(\bar{\Omega})$-norm. Indeed, there is no need to differentiate (1.2) in this case, and we obtain (2.11) directly in terms of the $C^{2+\alpha}(\bar{\Omega})$-topology. Then by the Schauder estimate, boundedness in the $\mathbb{R} \times C^{1+\alpha}$-norm implies boundedness in the $\mathbb{R} \times C^{2+\alpha}$-norm; also see [6].

\section{Positivity of the global continuUm}

Let

$P_{+}=\{u \in D, u>0$ on $\Omega, \partial u / \partial s>0$ on $\partial \Omega\}$, which is a positive cone in $D$; here $s$ denotes any direction that enters $\Omega$ transversally.

Obviously, $P_{+}$is open in $D$, and we claim that

$$
\Sigma_{0} \cap\left(\mathbb{R} \times P_{+}\right) \neq \varnothing .
$$

Indeed, $u_{0} \in P_{+}$by Lemma $H$ of [4]. By the Lyapunov-Schmidt reduction, any solution of $G(\lambda, u)=0$ near $\left(\lambda_{0}, 0\right)$ is of the form

$$
u=\varepsilon u_{0}+o(\varepsilon) \text { as } \varepsilon \rightarrow 0,
$$

where $(\lambda, \varepsilon)$ satisfies a scalar bifurcation equation

$$
\Phi(\lambda, \varepsilon)=0, \text { for which } \Phi_{\varepsilon}(\lambda, 0) \text { changes sign at } \lambda=\lambda_{0} \quad \text { (see [8]). }
$$

(As a matter of fact, the change of sign of $\Phi_{\varepsilon}(\lambda, 0)$ at $\lambda=\lambda_{0}$ is equivalent to an odd crossing number $\chi\left(\lambda_{0}\right)$ of the family $G_{u}(\lambda, 0)$ at $\left.\lambda=\lambda_{0}.\right)$ The Intermediate Value Theorem guarantees a (local) continuum $\{(\lambda, \varepsilon)\}$ near $\left(\lambda_{0}, 0\right)$ with $\varepsilon>0$ (and $\varepsilon<0$ ), and hence (3.2) follows from (3.3) and the assumption (2.4) on $u_{0}$.

Assume that $\Sigma_{0}$ leaves $\mathbb{R} \times P_{+}$. By the connectedness of $\Sigma_{0}$, there must be a solution $\left(\lambda^{*}, u^{*}\right) \in \Sigma_{0}$ belonging to the boundary of $\mathbb{R} \times P_{+}$: that is,

$$
\begin{aligned}
& u^{*} \geq 0 \text { on } \bar{\Omega}, \text { and there is at least one point } \mathbf{x}_{0} \in \bar{\Omega} \text { such that } \\
& u^{*}\left(\mathbf{x}_{0}\right)=\nabla u^{*}\left(\mathbf{x}_{0}\right)=0 \text {. }
\end{aligned}
$$


Observe that $\left(\lambda^{*}, u^{*}\right)$ solves the linear partial differential equation

$$
\begin{aligned}
L^{*} u= & {\left[\int_{0}^{1} F_{w_{i j}}\left(t \nabla^{2} u^{*}, t \nabla u^{*}, t u^{*}, \mathbf{x}, \lambda^{*}\right) d t\right] u_{x_{i} x_{j}} } \\
& +\left[\int_{0}^{1} F_{v_{i}}\left(t \nabla^{2} u^{*}, t \nabla u^{*}, t u^{*}, \mathbf{x}, \lambda^{*}\right) d t\right] u_{x_{i}} \\
& +\left[\int_{0}^{1} F_{u}\left(t \nabla^{2} u^{*}, t \nabla u^{*}, t u^{*}, \mathbf{x}, \lambda^{*}\right) d t\right] u=0 .
\end{aligned}
$$

As first observed by Serrin [11], the usual minimum principle for elliptic equations also holds without restricting the sign of the coefficient of the zeroorder term, provided that $u^{*} \geq 0$ (cf. also [4]). We employ this result, including the Hopf boundary lemma ([4, Lemma $\mathrm{H}])$, to conclude:

$$
\text { Condition (3.5) implies } u^{*} \equiv 0 \text {. }
$$

In other words,

$$
\text { if }\left(\lambda^{*}, u^{*}\right) \in \Sigma_{0} \text { is on the boundary of } \mathbb{R} \times P_{+} \text {then } u^{*} \equiv 0 \text {. }
$$

The same arguments employed above (maximum principle) imply the existence of a global negative part of $\Sigma_{0}$ that cannot leave $\mathbb{R} \times P_{-}$unless $u^{*} \equiv$ $0\left(P_{-} \equiv-P_{+}\right)$. We summarize:

Theorem 3.1. Assume the hypotheses of $\S 2$. Then the global continuum $\Sigma_{0} b i$ furcating at $\left(\lambda_{0}, 0\right)$ consists of a positive part in $P_{+}$and a negative part in $P_{-}$, each subject to the alternative (2.11).

Remark. Since we have not assumed oddness of $G(\lambda, \cdot)$, we do not exclude the possibility that the two parts behave differently.

Finally we note that

Corollary 3.1. Assume the hypotheses of $\S 2$. If $\lambda_{0} \in \mathbb{R}$ is the only parameter value for which (2.3) admits a positive eigenfunction, then $\Sigma_{0}$ is unbounded in $\mathbb{R} \times C^{4+\alpha}(\bar{\Omega})$.

\section{REFERENCES}

1. H. Amann, Fixed point equations and nonlinear eigenvalue problems in ordered banach spaces, SIAM Rev. 18 (1976), 620-709.

2. M. Crandall and P. H. Rabinowitz, Bifurcation from simple eigenvalues, J. Funct. Anal. 8 (1971), 321-340.

3. P. M. Fitzpatrick and J. Pejsachowicz, An extension of the Leray-Schauder degree for fully nonlinear elliptic problems, Proc. Sympos. Pure Math., vol. 45, Amer. Math. Soc., Providence, RI, 1986, pp. 425-438.

4. B. Gidas, W. M. Ni, and L. Nirenberg, Symmetry and related properties via the maximum principle, Comm. Math. Phys. 68 (1979), 209-243.

5. D. Gilbarg and N. S. Trudinger, Elliptic partial differential equations of second order, 2nd ed., Springer-Verlag, New York, 1983.

6. T. Healey and H. Kielhöfer, Symmetry and nodal properties in global bifurcation analysis of quasi-linear elliptic equations, Arch. Rational Mech. Anal. 113 (1991), 299-311.

7. P. Hess and T. Kato, On some linear and nonlinear eigenvalue problems with an indefinite weight function, Comm. Partial Differential Equations 5 (1986), 999-1030. 
8. H. Kielhöfer, Multiple eigenvalue bifurcation for Fredholm operators, J. Reine Angew. Math. 358 (1985), 104-124.

9. P. L. Lions, On the existence of positive solutions of semilinear elliptic equations, SIAM Rev. 24 (1982), 441-467.

10. P. H. Rabinowitz, Some aspects of nonlinear eigenvalue problems, Rocky Mountain J. Math. 3 (1973), 161-202.

11. J. Serrin, A symmetry problem in potential theory, Arch. Rational Mech. Anal. 43 (1971), 304-318.

12. E. Zeidler, Nonlinear functional analysis and its applications, Vol. I, Springer-Verlag, New York, 1986.

Department of Theoretical and Applied Mechanics and Center for Applied MatheMATICS, CORNELl UNIVERSITY, IthaCA, NEW YORK 14853-1503

InStitut für MAthematik, Universität Augsburg, Universitätsstrasse 8, 8900 AugsBURG, GERMANY 